\title{
DUAL SOLUTIONS FOR RADIATIVE MHD FORCED CONVECTIVE FLOW OF A NANOFLUID OVER A SLENDERING STRETCHING SHEET IN POROUS MEDIUM
}

\author{
C. Sulochana and N. Sandeep* \\ Department of Mathematics, Gulbarga University, Gulbarga-585106, Karnataka, India. \\ Email: math.sulochana@gmail.com, *Email: nsreddy.dr@gmail.com
}

\begin{abstract}
:
In this study we analyzed the magnetohydrodynamic forced convective flow of a nano fluid over a slendering stretching sheet in porous medium in presence of thermal radiation and slip effects. We presented dual solutions for no-slip and Navier slip conditions. Using self similarity transformation, the governing partial differential equations are transformed into nonlinear ordinary differential equations and solved numerically using BVP5C Matlab package. The effects of dimensionless governing parameters on velocity and temperature profiles of the flow are discussed with the help of graphs. Numerical computations are carried out and discussed for skin friction coefficient and local Nusselt number. We found an excellent agreement of the present results with the existed results under some special conditions. Results indicate that the dual solutions exist only for certain range of velocity slip parameter. It is also found that the heat transfer performance is high in presence of velocity slip effect.
\end{abstract}

Keywords: MHD, nano fluid, radiation, porous medium, slendering stretching sheet.

\section{Introduction}

The fluid flow and heat transfer over a stretching surface have various applications in engineering and sciences. Low thermal conductivity in convectional fluids like water, ethylene glycol, oil etc. encountered variety of problems in engineering electronic devices. To enhance the thermal conductivity of the base fluids (Choi, 1995) introduced a concept of nano fluid by immersing nanometer sized particles into the base fluids. Heat and mass transfer in MHD flow over a vertical stretching sheet in the presence of radiation was discussed by Rashidi et al. (2014). Prasad et al. (2010) studied MHD flow and heat transfer over a nonlinearly stretching sheet with variable fluid properties. Zaimi et al. (2014) discussed the flow of a nano fluid over a permeable stretching or shrinking sheet by using two phase model. Mohankrishna et al. (2014) discussed an unsteady natural convection flow of a nano fluid over an infinite vertical plate in presence of radiation and magnetic field effects and found that an increase in radiation parameter enhances the thermal boundary layer thickness.

Magnetohydrodynamic mixed convection flow of a micropolar fluid towards a shrinking vertical sheet with slip effects was illustrated by Das (2012). Hayat and Sajid (2007) presented an analytical solution for axisymmetric flow of a second grade fluid over a stretching sheet. Gupta et al. (2014) presented a finite element solution for mixed convection flow of a micropolar fluid over a porous shrinking sheet and concluded that an increase in the mixed convection parameter enhances the heat transfer rate. Mishra and Singh (2014) proposed a dual solution for mixed convection flow over a permeable shrinking cylinder with slip effects. Makinde and Aziz (2011) analyzed the boundary layer flow of a nano fluid over a stretching surface in presence of convective boundary conditions. Combined effects of MHD obliquely striking rheological fluid pasta stretching surface with partial slip conditions was discussed by Nadeem et al. (2015). Sandeep and Sulochana (2015) presented Dual solutions of MHD nano fluid flow over an exponentially stretching sheet in presence of radiation and generation or absorption and concluded that dual solutions exists only for certain range of suction or injection parameter. The slip effects on stagnation-point flow of a nano fluid over a stretching sheet with convective boundary conditions were discussed by Akbar et al. (2014). Raju et al. (2015) discussed the influence of thermal radiation and aligned magnetic field on the flow over a stretching sheet in the presence of thermo diffusion effects. Malvandi et al. (2014) studied the unsteady stagnation point flow of a nano fluid over a stretching sheet with slip effect. Rushikumar (2013) studied the MHD boundary layer flow on heat transfer over a stretching sheet in presence of slip effect. 
Momentum and heat transfer in $\mathrm{Cu}$-water nano fluid between stretching sheet and a porous surface in rotating system was analyzed by Sheikholeslami et al. (2012). Uddin et al. (2014) proposed a mathematical model for radiative MHD thermosolutal nano fluid convection slip flow in a porous medium. Ibrahim et al. (2013) discussed the MHD boundary layer flow of a nano fluid past a permeable stretching with velocity and thermal slip boundary conditions. Chaudhary and Jha (2008) studied the influence of chemical reaction on MHD micro polar fluid flow over a vertical plate in slip-flow regime. The effects of thermal radiation and chemical reaction on MHD convective flow over a permeable stretching surface with suction and heat generation was studied by Mohankrishna et al. (2015). Brewster (1972) discussed the thermal radiation effect on the heat transfer in convectional fluid flows. Effects of variable viscosity on non-Newtonian fluid flow in a porous medium with slip conditions were illustrated by Khan et al. (2013). Wang and Mujumdar (2007) discussed the heat transfer characteristics of nano fluids. Chakrabarti and Gupta (1979) have analyzed the MHD flow and heat transfer over a stretching sheet. Dual solutions for MHD boundary layer flow of nano fluids induced by a power-law stretching or shrinking permeable sheet in presence of viscous dissipation was studied by Dhanai et al. (2015). Gupta et al. (2014) presented a numerical solution for steady free convection flow of a power-law fluid from a heated spheroidal particle. MHD flow and heat transfer over a stretching surface with variable temperature was discussed by Devi and Thyagarajan (2006). The effect of radiation on the flow of a micropolar fluid over a nonlinearly stretching sheet was discussed by Babu et al. (2015). Khader and Megahed (2013) presented a numerical solution for boundary layer flow over a nonlinear stretching sheet in presence of slip conditions. Recently, Uddin et al. (2015) and Sandeep and Sulochana (2015) discussed the heat transfer characteristics of MHD flows through different channels.

In this study we discussed the magnetohydrodynamic forced convective flow of a nano fluid over a slendering stretching sheet in porous medium in presence of thermal radiation and slip effects. We presented dual solutions for no-slip and Navier slip conditions. Using self similarity transformation, the governing partial differential equations are transformed into nonlinear ordinary differential equations and solved numerically using bvp5c Matlab package. The effects of dimensionless governing parameters on velocity and temperature profiles of the flow are discussed with the help of graphs. Numerical computations are carried out and discussed for skin friction coefficient and local Nusselt number.

\section{Formulation of the Problem}

Consider a steady two-dimensional, incompressible, laminar, hydromagnetic flow of a nano fluid over a stretching sheet with non-uniform thickness in porous medium. No-slip and Navier slip conditions are taken into account. The sheet is along the $x$-axis direction and $y$-axis is normal to it as shown in Fig.1. A variable magnetic field $B(x)=B_{0}(x+a)^{-(1-n) / 2}, n \neq 1$ is applied to the flow, where $B_{0}$ is applied magnetic field strength and $a$ is the physical parameter related to stretching sheet. Magnetic Reynolds number is assumed to be very small so that the induced magnetic field is neglected. A non-uniform permeability $K(x)=k_{0}(x+a)^{1-n}, n \neq 1$ along with the thermal radiation effect is taken into account. Viscous dissipation effect is neglected in this study. It is assumed that the sheet is stretched with the velocity $u_{w}(x)=u_{0}(x+a)^{n}, n \neq 1$ and the wall temperature $T_{w}(x)=T_{\infty}+T_{0}(x+a)^{(1-n) / 2}, n \neq 1$. Since the sheet is non-uniform it is assumed that $y=A(x+a)^{(1-n) / 2}, n \neq 1$, where $A$ is the coefficient related to stretching sheet and chosen as a small constant to avoid the external pressure. At $n=1$ the problem refers flat stretching sheet case.

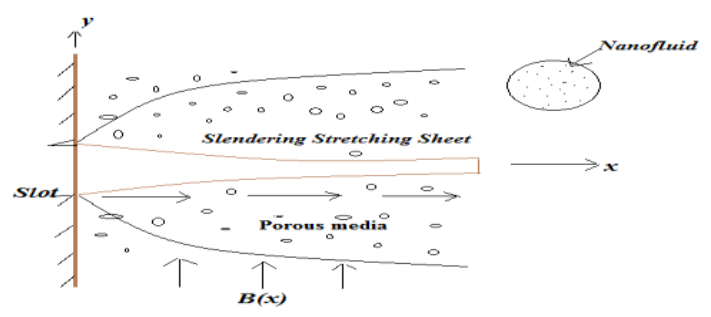

Fig.1: Physical model and coordinate system 
As per the above assumptions the governing boundary layer equations are given as follows: (Devi and Thiyagarajan, 2006)

$$
\begin{aligned}
& \frac{\partial u}{\partial x}+\frac{\partial v}{\partial y}=0, \\
& u \frac{\partial u}{\partial x}+v \frac{\partial u}{\partial y}=v_{f} \frac{\partial^{2} u}{\partial y^{2}}-\frac{\sigma B^{2}}{\rho_{f}} u-\frac{v_{f}}{K} u \\
& u \frac{\partial T}{\partial x}+v \frac{\partial T}{\partial y}=\alpha \frac{\partial^{2} T}{\partial y^{2}}-\frac{1}{\left(\rho c_{p}\right)_{f}} \frac{\partial q_{r}}{\partial y}
\end{aligned}
$$

The boundary conditions are as follows

$$
\left.\begin{array}{rr}
u(x, y)=u_{w}(x)+\delta_{1}^{*} \frac{\partial u}{\partial y}, v(x, y)=0, T(x, y)=T_{w}(x)+\delta_{2}^{*} \frac{\partial T}{\partial y}, & \text { at } y=0, \\
u(x, y) \rightarrow 0, T(x, y) \rightarrow T_{\infty}, n \neq 1 & \text { as } y \rightarrow \infty,
\end{array}\right\}
$$

where $u$ and $v$ are the velocity components along $x$ and $y$ directions respectively, $\rho_{f}$ is the density of the fluid, $v_{f}$ is the kinematic viscosity, $\sigma$ is the electrical conductivity, $T$ is the temperature of the fluid, $k$ is the thermal conductivity, $\alpha$ is the thermal diffusivity of the nano fluid, $\left(\rho c_{p}\right)_{f}$ is the specific heat capacitance, $\delta_{1}^{*}$ is the dimensional velocity slip parameter and $\delta_{2}^{*}$ is dimensional temperature jump parameter, these are given by $\delta_{1}^{*}=\left(\frac{2-b}{b}\right) \xi_{1}(x+a)^{(+n) \prime}, \delta_{2}^{*}=\left(\frac{2-c}{c}\right) \xi_{2}(x+a)^{(1-n) / 2}, \xi_{2}=\left(\frac{2 \lambda}{\lambda+1}\right) \frac{\xi_{1}}{\operatorname{Pr}}$, here $\xi_{1}, \xi_{2}$ are mean free paths and $\lambda$ is the ratio of specific heats, $b$ and $c$ respectively indicates Maxwell's reflection coefficient and thermal accommodation coefficient.

The radiative heat flux $q_{r}$ under Rosseland approximation has the form (Brewster, 1972)

$$
q_{r}=-\frac{4 \sigma^{*}}{3 k^{*}} \frac{\partial T^{4}}{\partial y},
$$

where $\sigma^{*}$ is the Stefan-Boltzmann constant and $k^{*}$ is the mean absorption coefficient. The temperature differences within the flow are assumed to be sufficiently small such that $T^{4}$ may be expressed as a linear function of temperature. Expanding $T^{4}$ using Taylor series and neglecting higher order terms yields $T^{4} \cong 4 T_{\infty}^{3} T-3 T_{\infty}^{4}$,

To convert the governing equations in to set of nonlinear ordinary differential equations we now introducing the following similarity transformation (Devi and Thiyagarajan, 2006)

$$
\psi(x, y)=\left(\frac{2 v_{f} u_{0}}{n+1}\right)^{0.5}(x+a)^{(n+1) / 2} f(\eta), n \neq 1, \quad \eta=\left(\frac{(n+1) u_{0}}{2 v_{f}}\right)^{0.5}(x+a)^{(n-1) / 2} y, n \neq 1,
$$

$\theta(\eta)=\left(T-T_{\infty}\right) /\left(T_{w}(x)-T_{\infty}\right)$,

where $\psi(x, y)$ is a stream function which satisfies the continuity equation (1) with

$$
u=\frac{\partial \psi}{\partial y} \text { and } v=-\frac{\partial \psi}{\partial x}
$$

Using Equations (5)-(8), Equations (2) and (3) can be reduced into the form

$$
f^{\prime \prime \prime}-\left(\frac{2 n}{n+1}\right) f^{\prime 2}+f f^{\prime \prime}-\left(M+K_{1}\right) f^{\prime}=0 \text {, }
$$


$\frac{1}{\operatorname{Pr}}\left(1+\frac{4}{3} R\right) \theta^{\prime \prime}-\left(\frac{1-n}{1+n}\right) f^{\prime} \theta+f \theta^{\prime}=0$

with the transformed boundary conditions

$\left.\begin{array}{l}f(\gamma)=\gamma\left(\frac{1-n}{1+n}\right)\left[1+\delta_{1} f^{\prime \prime}(0)\right], f^{\prime}(\gamma)=\left[1+\delta_{1} f^{\prime \prime}(0)\right], \\ \theta(\gamma)=\left[1+\delta_{2} \theta^{\prime}(0)\right], f^{\prime}(\infty) \rightarrow 0, \theta(\infty) \rightarrow 0, n \neq 1,\end{array}\right\}$

where $\gamma=A\left(\frac{(n+1) u_{0}}{2 v_{f}}\right)^{0.5}, \delta_{1}=\left(\frac{2-b}{b}\right) \xi_{1}\left(\frac{(n+1) u_{0}}{2 v_{f}}\right)^{0.5}, \delta_{2}=\left(\frac{2-c}{c}\right) \xi_{2}\left(\frac{(n+1) u_{0}}{2 v_{f}}\right)^{0.5}$,

Here equations (9) and (10) are nonlinear ordinary differential equations in the domain $[\gamma, \infty)$.

For numerical computation we transformed the domain $[\gamma, \infty)$ into $[0, \infty)$ by defining $F(\xi)=F(\eta-\gamma)=f(\eta)$ and $\Theta(\xi)=\Theta(\eta-\gamma)=\theta(\eta)$. Now the similarity equations become

$F^{\prime \prime}-\left(\frac{2 n}{n+1}\right) F^{\prime 2}+F F^{\prime \prime}-\left(M+K_{1}\right) F^{\prime}=0$,

$\frac{1}{\operatorname{Pr}}\left(1+\frac{4}{3} R\right) \Theta{ }^{\prime \prime}-\left(\frac{1-n}{1+n}\right) F^{\prime} \Theta+F \Theta^{\prime}=0$,

with the boundary conditions

$\left.\begin{array}{l}F(0)=\gamma\left(\frac{1-n}{1+n}\right)\left[1+\delta_{1} F^{\prime \prime}(0)\right], F^{\prime}(0)=\left[1+\delta_{1} F^{\prime \prime}(0)\right], \\ \Theta(0)=\left[1+\delta_{2} \Theta^{\prime}(0)\right], F^{\prime}(\infty) \rightarrow 0, \Theta(\infty) \rightarrow 0, n \neq 1,\end{array}\right\}$

where the prime indicates the differentiation with respect to $\xi$. $M=2 \sigma B_{0}^{2} / \rho_{f} u_{0}(1+n)$ is the magnetic field parameter, $K_{1}=2 v_{f} / k_{0} u_{0}(n+1)$ is the porosity parameter, $\operatorname{Pr}=v_{f} / \alpha$ is the Prandtl number, $R=4 \sigma T_{\infty}^{3} / k k^{*}$ is the radiation parameter, $n$ is the velocity power index parameter, $\gamma$ is the wall thickness parameter, $\delta_{1}$ is the non-dimensional velocity slip parameter and $\delta_{2}$ is the non-dimensional temperature jump parameter.

For engineering interest the shear stress coefficient or friction factor $\left(C_{f}\right)$ and local Nusselt number $\left(N u_{x}\right)$ are given by

$\operatorname{Re}_{x}^{0.5} C_{f}=2\left(\frac{n+1}{2}\right)^{0.5} F^{\prime \prime}(0)$,
$\operatorname{Re}_{x}^{-0.5} N u_{x}=-\left(\frac{n+1}{2}\right)^{0.5} \Theta^{\prime}(0)$,

where $\operatorname{Re}_{x}=\frac{u_{w}(x+a)}{v_{f}}$ is the local Reynolds number.

\section{Results and Discussion}

The nonlinear ordinary differential equations (12) and (13) subjected to the boundary conditions (14) are solved numerically using BVP5C Matlab package. The effects of dimensionless governing parameters namely magnetic field parameter $(M)$, Radiation parameter $(R)$, porosity parameter $\left(K_{1}\right)$, velocity power law index ( $n)$, Prandtl number $(\operatorname{Pr})$, temperature jump parameter $\left(\delta_{2}\right)$ and wall thickness parameter $(\gamma)$ on the velocity and temperature profiles are discussed. Also, found the influence of non-dimensional governing parameters on 
the skin friction coefficient and local Nusselt number. For numerical computations we considered $n=R=K_{1}=\delta_{2}=0.5, \operatorname{Pr}=7, M=1, \gamma=0.2,0.1 \leq \delta_{1} \leq 1$. These values are kept as common in entire study except the varied values as shown in the respective figures and tables.

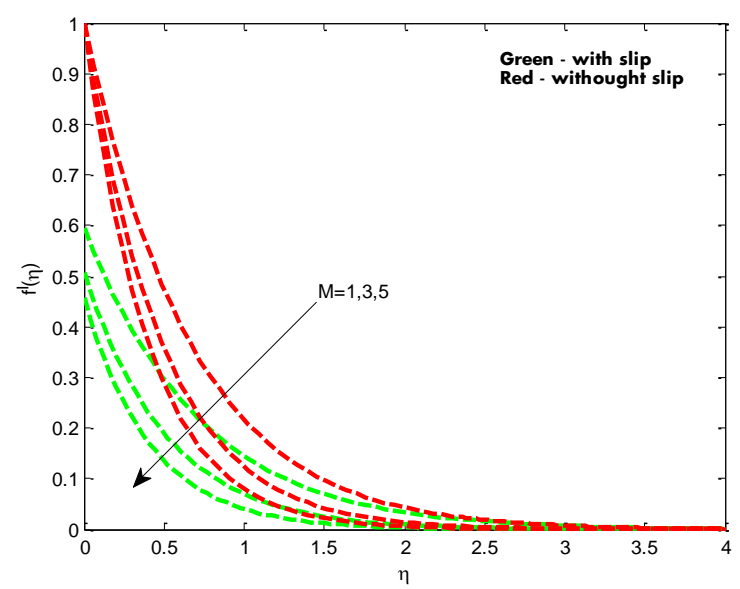

Fig. 2: Velocity filed for various values of magnetic field parameter $M$

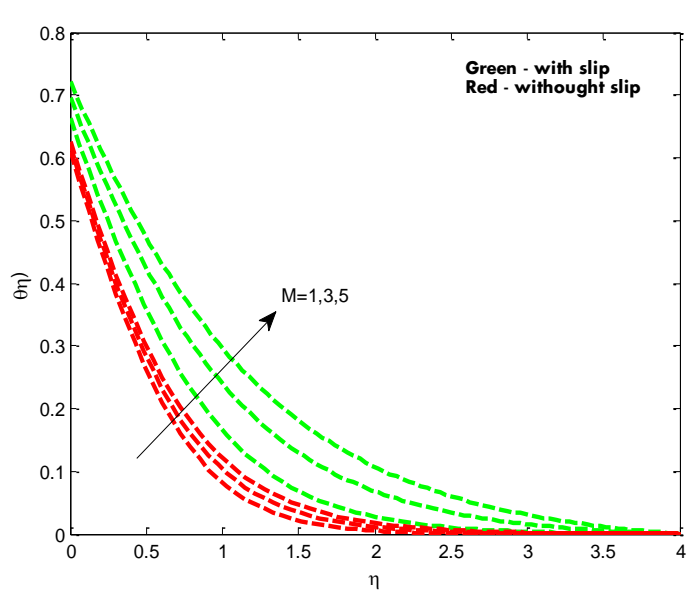

Fig. 3: Temperature filed for various values of magnetic field parameter $M$

Figs. 2 and 3 depict the effect of magnetic field parameter on velocity and temperature profiles of the flow for slip and no-slip conditions. It is evident that for higher values of magnetic field parameter we observed depreciation in velocity profiles and hike in the temperature profiles of the flow. It is observed that in presence of slip effect the velocity boundary layer become thin with the increase in magnetic field parameter. Since the fluid is electrically conducting, a raise in the value of magnetic field parameter improves the interaction between electric and magnetic field, which causes to boost the Lorentz's force. This force works opposite to the flow and reduces the momentum boundary layer thickness and enhances the thermal boundary layer thickness.

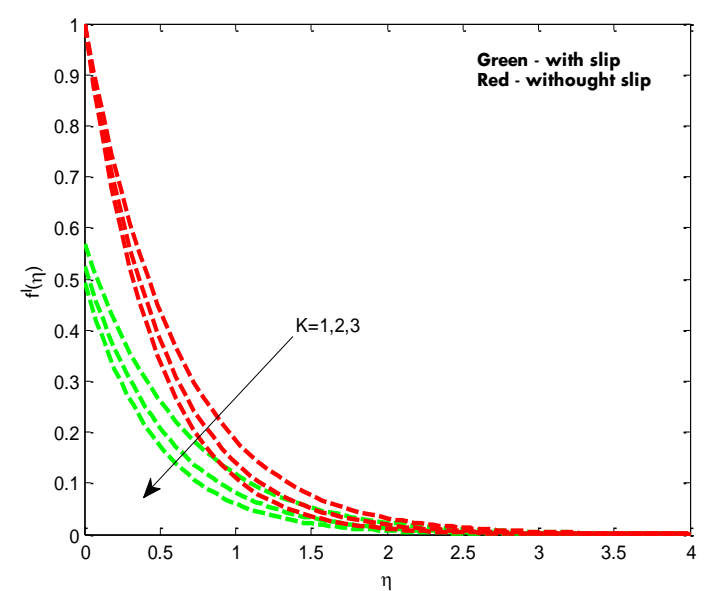

Fig. 4: Velocity filed for various values of porosity parameter $K$

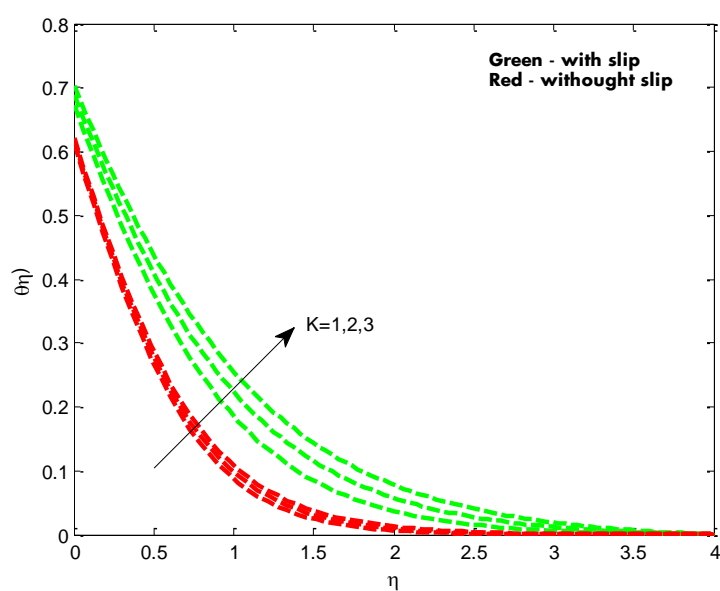

Fig. 5: Temperature filed for various values of porosity parameter $K$

Figs. 4 and 5 illustrate the effect of porosity parameter on velocity and temperature profiles of the flow for slip and no-slip conditions. With the increase in the porosity parameter we have seen the similar type of results as we observed in the magnetic field parameter case. This is due to the fact that an increase in the porosity parameter widens the holes of porous layer, these causes to reduce the momentum boundary layer thickness. In the other hand a raise in the porosity parameter generates and releases the internal heat energy to the flow. Due to this reason we noticed a hike in the temperature profiles of the flow. Figs. 6 and 7 display the influence of power law index on velocity and temperature profiles of the flow for slip and no-slip conditions. With the increase in the power law index we observed a raise in the temperature and reduction in the velocity profiles of 
the flow. An increase in the power law index reduced the thickness of the sheet. This causes to improve the thermal conductivity of the flow and reduce the momentum boundary layer thickness.

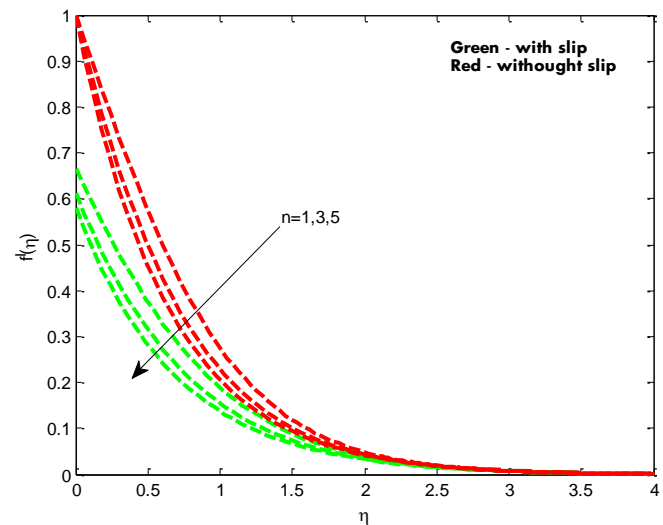

Fig. 6: Velocity filed for various values of power law index parameter $n$

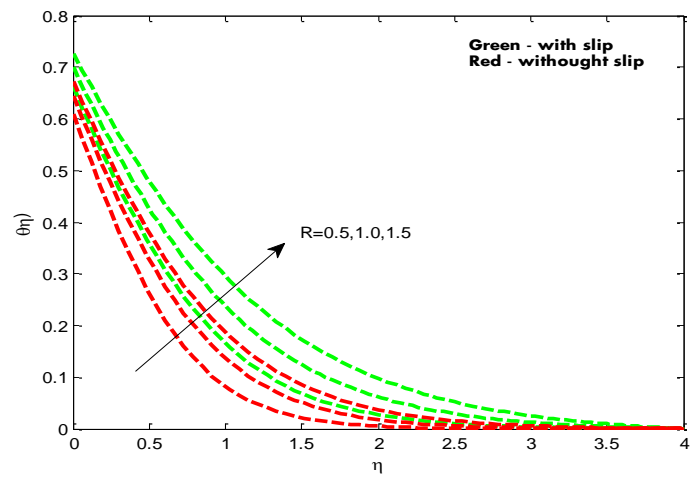

Fig. 8: Temperature filed for various values of radiation parameter $R$

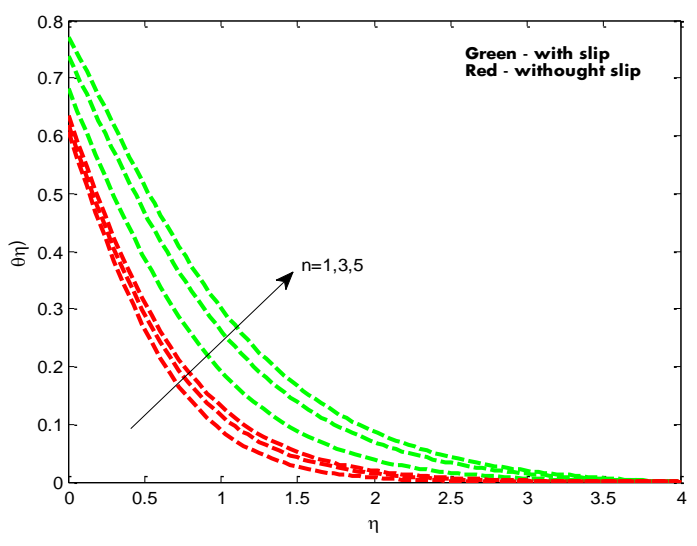

Fig. 7: Temperature filed for various values of power law index parameter $n$

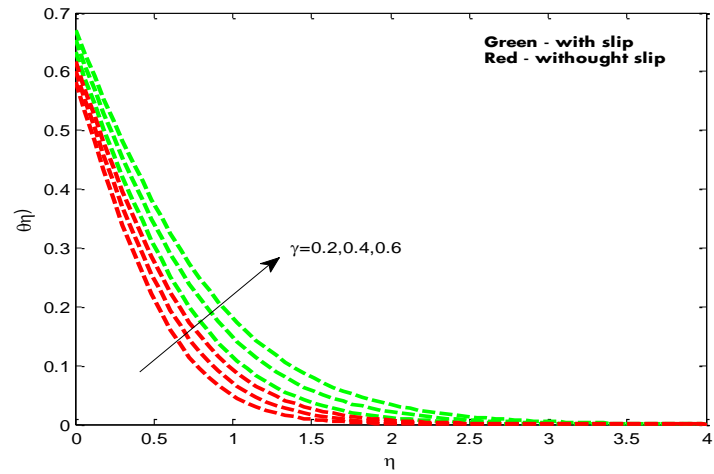

Fig. 9: Temperature filed for various values of wall thickness parameter $\gamma$

Figs. 8 and 9 respectively depict the effect of radiation and wall thickness parameters on the temperature profiles of the flow for slip and no-slip conditions. It is evident that with the increase in the radiation and wall thickness parameters we observed a raise in the temperature profiles of the flow. Generally, increasing in the radiation parameter releases the heat energy to the flow. An increase in the wall thickness causes to enhance the thermal conductivity of the flow, which causes to develop the thermal boundary layer thickness. Figs. 10 and 11 present the effect of Prandtl number and the non-dimensional temperature jump parameter on temperature profiles of the flow. It is clear that with the increase in Prandtl number and temperature jump parameter we noticed depreciation in the temperature profiles of the flow. It is the expected result that an increase in the nondimensional temperature jump parameter strengthens the thermal accommodation coefficient, which causes to reduce the thermal diffusion towards the flow along with this the thermal boundary layer become thinner with the increase in the non-dimensional temperature jump parameter.

Table 1: Comparison of the values of $F^{\prime \prime}(0)$ when $R=M=\delta_{2}=K_{1}=0, n=0.5$

\begin{tabular}{|cccc|}
\hline$\gamma$ & $\delta_{1}$ & $\begin{array}{c}\text { Khader and Megahed } \\
(2013)\end{array}$ & Present study \\
\hline 0.2 & 0 & -0.924828 & -0.9248278 \\
\hline 0.25 & 0.2 & -0.733395 & -0.7333951 \\
\hline 0.5 & 0.2 & -0.759570 & -0.7595702 \\
\hline
\end{tabular}

Table 1 shows the comparison of the present results with the existed results of Khader and Megahed (2013). We found an excellent agreement with the existed results under some special conditions. This shows the validity of the present work and the numerical technique we used in this study. Table 2 depicts the effects of various non- 
dimensional governing parameters on friction factor and local Nusselt number. It is evident that an increase in the values of magnetic field parameter, porosity parameter and power law index parameter depreciates the friction factor and heat transfer rate. A raise in the values of radiation parameter, wall thickness parameter and temperature jump parameter causes to decrease the Nusselt number. But these parameters do not shown significant variation in friction factor. Prandtl number have tendency to enhance the heat transfer rate.

Table. 2: Variation in $F^{\prime \prime}(0)$, and $-\Theta^{\prime}(0)$ for different non-dimensional parameters with slip effect

\begin{tabular}{|c|c|c|c|c|c|c|c|c|}
\hline$M$ & $K$ & $n$ & $R$ & $\gamma$ & $\operatorname{Pr}$ & $\delta_{2}$ & $F^{\prime \prime}(0)$ & $-\Theta^{\prime}(0)$ \\
\hline 1 & & & & & & & -0.808260 & 0.675280 \\
\hline 3 & & & & & & & -0.983387 & 0.607266 \\
\hline 5 & & & & & & & -1.087687 & 0.556773 \\
\hline & 1 & & & & & & -0.863490 & 0.656167 \\
\hline & 2 & & & & & & -0.949033 & 0.622329 \\
\hline & 3 & & & & & & -1.013748 & 0.593273 \\
\hline & & 1 & & & & & -0.838097 & 0.637846 \\
\hline & & 3 & & & & & -0.877431 & 0.525213 \\
\hline & & 5 & & & & & -0.889348 & 0.457391 \\
\hline & & & 0.5 & & & & -0.808260 & 0.675280 \\
\hline & & & 1.0 & & & & -0.808260 & 0.600469 \\
\hline & & & 1.5 & & & & -0.808260 & 0.545364 \\
\hline & & & & 0.2 & & & -0.808260 & 0.734445 \\
\hline & & & & 0.4 & & & -0.808260 & 0.693534 \\
\hline & & & & 0.6 & & & -0.808260 & 0.658276 \\
\hline & & & & & 6 & & -0.808260 & 0.641069 \\
\hline & & & & & 7 & & -0.808260 & 0.675280 \\
\hline & & & & & 8 & & -0.808260 & 0.704455 \\
\hline & & & & & & 0.2 & -0.808260 & 0.846835 \\
\hline & & & & & & 0.4 & -0.808260 & 0.724182 \\
\hline & & & & & & 0.6 & -0.808260 & 0.632564 \\
\hline
\end{tabular}

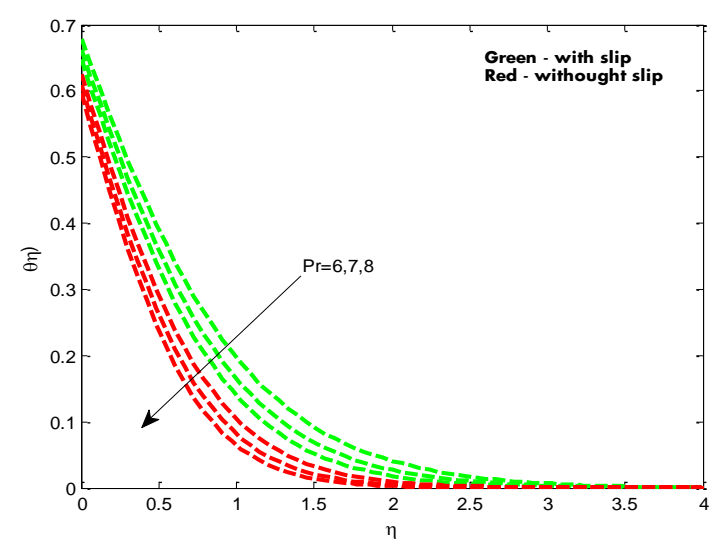

Fig. 10: Temperature filed for various values of Prandtl number $\operatorname{Pr}$

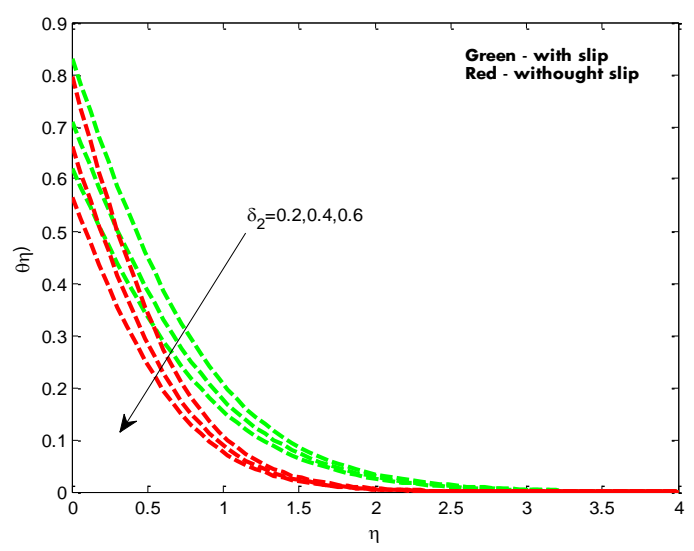

Fig. 11: Temperature filed for various values of temperature jump parameter $\delta_{2}$

\section{Conclusions}

This study presents a numerical solution for the magneto-hydrodynamic forced convective flow of a nano fluid over a slendering stretching sheet in porous medium in presence of thermal radiation and slip effects. We 
presented dual solutions for no-slip and Navier slip conditions. Using self-similarity transformation, the governing partial differential equations are transformed into nonlinear ordinary differential equations and solved numerically using BVP5C Matlab package. The effects of dimensionless governing parameters on velocity and temperature profiles of the flow are discussed with the help of graphs. Numerical computations are carried out and discussed for skin friction coefficient and local Nusselt number. Conclusions of the present study are made as follows:

- Dual solutions exist only for certain range of velocity slip parameter.

- Thermal boundary layer thicknesses become high in presence of velocity slip.

- An increase in the magnetic field parameter reduces the friction factor and local Nusselt number.

- Radiation parameter does not influence the friction factor. But for higher values of radiation parameters we noticed a hike in the temperature profiles of the flow.

- Increases in power law index depreciate the momentum and enhance the thermal boundary layer thicknesses.

- An increase in the wall thickness causes to enhance the temperature profiles of the flow.

- Magneticfiled have tendency to control the flow.

\section{Acknowledgements}

Authors acknowledge the UGC for financial support under the UGC Dr. D. S. Kothari Post-Doctoral Fellowship Scheme (No.F.4-2/2006 (BSR)/MA/13-14/0026).

\section{References}

Akbar, N. S., Khan, Z. H., Nadeem, S. (2014): The combined effects of slip and convective boundary conditions on stagnation-point flow of CNT suspended nano fluid over a stretching sheet, Journal of Molecular Liquids, Vol. 196, pp. 21-25. http://dx.doi.org/10.1016/j.molliq.2014.03.006

Babu, M. J., Gupta, R., Sandeep, N. (2015): Effect of radiation and viscous dissipation on stagnation-point flow of a micropolar fluid over a nonlinearly stretching surface with suction/injection, Journal of Basic and Applied Research International, Vol. 7, No. 2, pp. 73-82.

Brewster, M.Q. (1972): Thermal radiative transfer properties, John Wiley and Sons.

Chakrabarti, A., Gupta, A. S. (1979): Hydromagnetic flow and heat transfer over a stretching sheet, Quarterly of Applied Mathematics, Vol. 37, pp. 73-98.

Chaudhary, R. C., Jha, A. K. (2008): Effects of chemical reactions on MHD micropolar fluid flow past a vertical plate in slip flow regime, Applied Mathematics and Mechanics, Vol. 29, No. 9, pp. 1179-1194.

http://dx.doi.org/10.1007/s10483-008-0907-x

Choi, S. U. S. (1995): Enhancing thermal conductivity of fluids with nano particles, The proceedings of the 1995 ASME International Mechanical Engineering Congress and Exposition, ASME, San Francisco, Vol.66, pp. 99-105.

Das, K. (2012): Slip effects on MHD mixed convection stagnation point flow of a micropolar fluid towards a shrinking vertical sheet, Computers and Mathematics with Applications, Vol. 63, pp.255-267.

http://dx.doi.org/10.1016/j.camwa.2011.11.018

Devi, S. P. A., Thiyagarajan, M. (2006): Steady nonlinear hydromagnetic flow and heat transfer over a stretching surface of variable temperature, Heat and Mass Transfer, Vol. 42, No. 8, pp. 671-677.

http://dx.doi.org/10.1007/s00231-005-0640-y

Dhanai, R., Rana, P., Kumar, L. (2015): Multiple solutions of MHD boundary layer flow and heat transfer behavior of nano fluids induced by a power-law stretching/shrinking permeable sheet with viscous dissipation, Power Technology, Vol. 273, pp.62-70. http://dx.doi.org/10.1016/j.powtec.2014.12.035

Gupta, D., Kumar, L., Singh, B. (2014): Finite element solution of unsteady mixed convection flow of micropolar fluid over a porous shrinking sheet, The Scientific World Journal, Vol. 2014, ID 362351, 11 pages, http://dx.doi.org/10.1155/2014/362351, http://dx.doi.org/10.1155/2014/362351 
Gupta, A. K., Sasmal, C., Sairamu, M., Chhabra, R. P. (2014): Laminar and steady free convection in power-law fluids from a heated spheroidal particle: A numerical study, International Journal of Heat and mass transfer, Vol. 75, pp.592-609. http://dx.doi.org/10.1016/j.ijheatmasstransfer.2014.04.008

Hayat, T., Sajid, M. (2007): Analytic solution for axisymmetric flow and heat transfer of a second grade fluid past a stretching sheet. International Journal of Heat Mass Transfer, Vo. 50, pp.75-84.

http://dx.doi.org/10.1016/j.ijheatmasstransfer.2006.06.045

Ibrahim, W., Shankar, B. (2013): MHD boundary layer flow and heat transfer of a Nano fluid past a permeable stretching with velocity, thermal and solutal slip boundary conditions, Computers \& Fluids, Vol. 75, No. 20, pp:1-10. http://dx.doi.org/10.1016/j.compfluid.2013.01.014

Khader, M., Megahed, A. M. (2013): Numerical solution for boundary layer flow due to a nonlinearly stretching sheet with variable thickness and slip velocity, The European Physical Journal Plus, Vol. 128, pp. 100-108.

http://dx.doi.org/10.1140/epjp/i2013-13100-7

Khan, A.A., Ellahi, R., Usman, M. (2013): Effects of variable viscosity on the flow of non-Newtonian fluid through a porous medium in an inclined channel with slip conditions, Journal of Porous media, Vol. 16, No. 1, pp. 59-67. http://dx.doi.org/10.1615/JPorMedia.v16.i1.60

Makinde, O.D., Aziz, A. (2011): Boundary layer flow of a nano fluid past a stretching sheet with convective boundary condition, International Journal of Thermal Science, Vol. 50, pp. 1326-1332.

http://dx.doi.org/10.1016/j.ijthermalsci.2011.02.019

Malvandi, A., Hedayati, F., Ganji, D. D. (2014): Slip effects on unsteady stagnation point flow of a nano fluid over a stretching sheet, Powder Technology, Vol. 253, pp. 377-384.

http://dx.doi.org/10.1016/j.powtec.2013.11.049

Mishra, U., Singh, G. (2014): Dual solutions of mixed convection flow with momentum and thermal slip flow over a permeable shrinking cylinder, Computers \& Fluids, Vol. 93, pp. 107-115.

http://dx.doi.org/10.1016/j.compfluid.2014.01.012

Mohankrishna, P., Sugunamma, V., Sandeep, N. (2014): Radiation and magnetic field effects on unsteady natural convection flow of a nano fluid past an infinite vertical plate with heat source, Chemical and Process Engineering Research, Vol. 25, pp.39-52.

Mohankrishna, P., Sandeep, N., Sugunamma, V. (2015): Effects of radiation and chemical reaction on MHD convective flow over a permeable stretching surface with suction and heat generation, Walaliak Journal of Science and Technology, Vol. 12, No. 9, pp. 831-847.

Nadeem, S., Mehmood, R., Akbar, N. S. (2015): Combined effects of magnetic field and partial slip on obliquely striking rheological fluid over a stretching surface, Journal of Magnetism and Magnetic Materials, Vol. 378, pp. 457-462. http://dx.doi.org/10.1016/j.jmmm.2014.11.043

Prasad, K. V., Vajravelu, K., Datti, P. S. (2010): The effect of variable fluid properties on the hydromagnetic flow and heat transfer over a nonlinearly stretching sheet, International Journal of Thermal Science, Vol. 49, pp. 603-610. http://dx.doi.org/10.1016/j.ijthermalsci.2009.08.005

Raju, C. S. K., Sandeep, N., Sulochana, C., Sugunamma, V., Jayachandra Babu, M. (2015): Radiation, inclined magnetic field and cross-diffusion effects on flow over a stretching surface, Journal of Nigerian Mathematical Society, Vol. 34, No. 1, pp. 169-180. http://dx.doi.org/10.1016/j.jnnms.2015.02.003

Rashidi, M. M., Rostami, B., Freidoonimehr, N., Abbasbandy, S. (2014): Free convective heat and mass transfer for MHD fluid flow over a permeable vertical stretching sheet in the presence of radiation and buoyancy effects, Ain Shams Engineering Journal, Vol. 5, pp. 901-912. http://dx.doi.org/10.1016/j.asej.2014.02.007

Rushi Kumar, B. (2013): MHD boundary layer flow on heat and mass transfer over a stretching sheet with slip effect, Journal of Naval Architecture and Marine Engineering, Vol. 0, No. 2, pp. 16-26.

Sandeep, N., Sulochana, C. (2015): Dual solutions of radiative MHD nano fluid flow over an exponentially stretching sheet with heat generation/absorption, Applied Nano Science, Vol. 5.

http://dx.doi.org/10.1007/s13204-015-0420-z, http://dx.doi.org/10.1007/s13204-015-0420-z

Sandeep, N, Sulochana, C. (2015): MHD flow of dusty nano fluid over a stretching surface with volume fraction of dust particles, Ain Shams Engineering Journal. http://dx.doi.org/10.1016/j.asej.2015.05.015,

http://dx.doi.org/10.1016/j.asej.2015.05.015 
Sheikholeslami, M., Ashorynejad, H. R., Domairry, G., Hashim, I. (2012): Flow and heat transfer of Cu-water nano fluid between stretching sheet and a porous surface in rotating system, Journal of Applied Mathematics, vol. 2012, Article ID: 421320. http://dx.doi.org/10.1155/2012/421320

Uddin, M. J., Beg, O. A., Ismail, A. I. M. (2014): Mathematical modeling of radiative hyderomagnetic thermosolutal nano fluid convection slips flow in saturated porous media, Mathematical Problems in Engineering, Vol.2014, pp.1-11.

Uddin, M. N., Farhana, A., Alim, M. A. (2015): Numerical study of magneto-hydrodynamic (MHD) mixed convection flow in a lid-driven triangular cavity, Journal of Naval Architecture and Marine Engineering, Vol. 11, No. 1, http://dx.doi.org/10.3329/jname.v12i1.12910

http://dx.doi.org/10.3329/jname.v12i1.12910

Wang, X.Q., Mujumdar, A.S. (2007): Heat transfer characteristics of nano fluids: a review, International Journal of Thermal Sciences, Vol. 46, pp.1-19.

http://dx.doi.org/10.1016/j.ijthermalsci.2006.06.010

Zaimi, K., Ishak, A., Pop, I. (2014): Flow past a permeable stretching/shrinking sheet in a nano fluid using two phase model, Plos one, Vol. 9, No.11, ID: e111743. 